\title{
Activity and Safety of Standard and Prolonged Capecitabine/Temozolomide Administration in Patients with Advanced Neuroendocrine Neoplasms
}

\author{
Eleftherios Chatzellis ${ }^{a, b} \quad$ Anna Angelousi ${ }^{a}$ Kosmas Daskalakis ${ }^{a, c}$ Marina Tsolia \\ Krystallenia I. Alexandraki ${ }^{a}$ Ewa Wachuład $^{d}$ Amichay Meirovitz $^{\mathrm{e}}$ Ofra Maimon ${ }^{\mathrm{e}}$ \\ Simona Grozinsky-Glasberg ${ }^{f}$ David Gross ${ }^{f}$ Beata Kos-Kudłag Anna Koumarianou ${ }^{\text {h }}$ \\ Gregory Kaltsas ${ }^{a}$

\begin{abstract}
a 1st Department of Propaedeutic Internal Medicine, National and Kapodistrian University of Athens, Athens, Sweden; ${ }^{d}$ Department of Clinical Oncology and Radiotherapy, Medical University of Silesia, Katowice, Poland; e Oncology Department and Radiation Therapy Unit, Hadassah-Hebrew University Medical Center, Jerusalem,

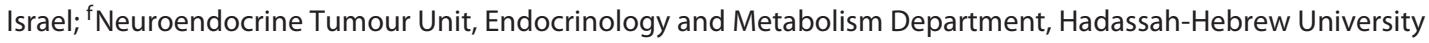
Medical Center, Jerusalem, Israel; ${ }^{9}$ Department of Endocrinology and Neuroendocrine Neoplasms, Department of Endocrinology and Pathophysiology, Medical University of Silesia, Katowice, Poland; ${ }^{\text {h}}$ Fourth Department of Internal Medicine, Hematology-Oncology Unit, Attikon University General Hospital, National and Kapodistrian
\end{abstract} \\ Greece; ${ }^{\text {b }} 251$ HAF and VA Hospital, Athens, Greece; ' Department of Surgical Sciences, Uppsala University, Uppsala, \\ University of Athens, Athens, Greece
}

\section{Keywords}

Temozolomide $\cdot$ Capecitabine $\cdot$ Capecitabine and temozolomide combination · TEMCAP · Neuroendocrine neoplasms $\cdot$ Neuroendocrine tumors

\footnotetext{
Abstract

Background: Capecitabine and temozolomide combination (CAPTEM) is associated with high response rates in patients with advanced neuroendocrine neoplasms (NENs). We evaluated the real-world activity and safety of CAPTEM from 3 NEN centers. Methods: Clinicopathological characteristics and outcomes of patients treated with CAPTEM for bulky or progressive disease (PD) were retrospectively analyzed. Results: Seventy-nine patients with gastroenteropancreatic (grades 1-2 $[n=38]$, grade $3[n=24])$ and lung/thymic $(n=17)$ NENs were
}

included. Median treatment duration was 12.1 months (range 0.6-55.6). Overall, partial responses (PRs) occurred in 23 (29.1\%), stable (SD) in 24 (30.4\%), and PD in 28 (35.4\%) patients. Median progression-free survival (PFS) and overall survival (OS) were 10.1 (6-14.2) and 102.9 months (43.3-162.5), respectively. On univariate analysis, NENs naive to chemotherapy and low Ki67 were associated with favorable responses (partial response $[\mathrm{PR}]+\mathrm{SD} ; p=0.011$ and 0.045$)$, PFS ( $p<$ 0.0001 and 0.002 ) and $O S(p=0.005$ and 0.001$)$. Primary site (pancreas and lung/thymus) was also a significant prognostic factor for PFS $(p<0.0001)$ and OS $(p<0.0001)$. On multivariate analysis, gastrointestinal and unknown primary NENs (hazard

E.C., A.A., and K.D. contributed equally to the preparation of the manuscript.

\section{KARGER}

(c) 2019 S. Karger AG, Basel

E-Mail karger@karger.com

www.karger.com/nen
Eleftherios Chatzellis

251 HAF and VA Hospital, Endocrinology Department

3 Kanelopoulou Avenue

GR-11525 Athens (Greece)

E-Mail e.chatzellis@endo.gr 
ratio [HR] $0.3,95 \% \mathrm{Cl} 0.1-0.8, p=0.009$ and $p=0.018$ ) and prior surgery (HR 2.4, 95\% Cl 11-4.9, $p=0.021$ ) were independent prognostic factors for PFS. Ki-67 was a poor predictor for favorable response in receiver operating characteristic analysis (area under the curve 0.678 ). Safety analysis of CAPTEM indicated rare events of serious (grades 3-4) toxicities $(n=4)$ and low discontinuation rates $(n=8)$ even in patients with prolonged administration (>12 months). Conclusions: CAPTEM treatment can be an effective and safe treatment even after prolonged administration for patients with NENs of various sites and Ki67 labeling index, associated with significant favorable responses and PFS.

(c) 2019 S. Karger AG, Basel

\section{Introduction}

Neuroendocrine neoplasms (NENs) arise from cells of the diffuse neuroendocrine system commonly encountered in the gastrointestinal (GI) tract and the lungs. Their prevalence has substantially increased during the last 2 decades [1]. Although frequently diagnosed at an advanced stage with distant metastases, they are considered to have a great variability in clinical behavior and prognosis based on site of origin, differentiation, and proliferation indices such as mitotic count and the ki-67 labeling index (LI) [2].

Gastroenteropancreatic NENs are categorized based on cell morphology and proliferation index as well-differentiated neuroendocrine tumors (WD-NETs) including grade 1 (G1: Ki67 LI <3\%) and grade 2 tumors (G2: Ki67 LI $3-20 \%)$ and as poorly differentiated neuroendocrine carcinomas including grade 3 tumors (G3: Ki67 LI > 20\%) [3]. In a 2017 update [4], G3 pancreatic neuroendocrine neoplasms (panNENs) were further divided in WD G3 panNETs (Ki67 LI $>20 \%$ but usually $<50 \%$ ) and PD G3 panNECs (Ki67 LI usually >50\%). Lung NENs are classified based on the number of mitoses and the presence of necrosis, encompassing well-differentiated tumors with low (typical carcinoids) and intermediate (atypical carcinoids) malignant potential, together with the more aggressive poorly differentiated small cell and large cell neuroendocrine carcinomas [5].

In recent years, a variety of therapeutic agents including somatostatin analogs, the multikinase inhibitor sunitinib and the mammalian target of rapamycin inhibitor everolimus have been approved as medical therapies for locally advanced, metastatic, or inoperable WD-NENs [6-10]. These agents, despite progression-free survival (PFS) prolongation, a well-known, relatively mild sideeffect profile and ease of administration, are not associ- ated with significant objective response rates (ORRs), but mostly delay tumor growth [11]. Streptozotocin-based chemotherapy has proven efficacious for a subset of WDpanNENs, while platinum-based chemotherapy regimens remain the cornerstone of treatment for poorly differentiated neuroendocrine carcinomas; however, both are associated with significant toxicity [12].

Temozolomide (TEM), a less toxic oral derivative of intravenous dacarbazine, is a second-generation alkylating agent acting though methylation of the DNA and triggering cell death by apoptosis [13]. This action is not cell cycle specific and can consequently inhibit all stages of tumor cell growth, even in tumors with low proliferation dynamics such as WD-NENs.

In this sense, TEM has been investigated as a monotherapy or in combination with other drugs (capecitabine, bevacizumab, thalidomide, everolimus) in low and intermediate grade NENs showing significant tumor responses in pancreatic and thoracic NENs, but has also been evaluatedin G3 tumors [14-17]. The combination of capecitabine with temozolomide (CAPTEM) is a commonly employed regimen, supported by in vitro evidence showing a synergistic effect in a sequence-specific manner. It is believed that pretreatment with capecitabine depletes the DNA repair enzyme $\mathrm{O}^{6}$-methylguanine DNA methyltransferase (MGMT), thereby potentiating the effect of temozolomide $[18,19]$. The role of MGMT as a response predictor, either assessed with immunohistochemistry (IHC) or with molecular analysis of MGMT gene promoter methylation by polymerase chain reaction, is still under investigation since conflicting data have been reported [20, 21]. In clinical studies, CAPTEM has been associated with significant ORR in either chemotherapy-naive or heavily pretreated patients mainly with panNENs, although gastrointestinal neuroendocrine neoplasms (GI-NENs) from other sites and non-gastroenteropancreatic-NENs primaries may also be responsive [22-25].

However, until now, data for TEM in NEN patients are derived from either observational retrospective series or small, single-institution, prospective phase II studies using a variety of different combinations and TEM dose schedules [14-16, 22, 26-28]. Currently, there is no published data comparing the efficacy of CAPTEM with other TEM-based combinations or with TEM alone (NCT01824875 and NCT03279601 are under way), nor are there data from prospective randomized trials comparing this regimen with streptozotocin- or platinumbased therapies. Several open questions regarding the timing of therapy, optimal schedule, duration of treatment, and long-term toxicity remain unanswered. 
Considering the previously demonstrated activity of the CAPTEM combination in patients with advanced NENs, we sought to document the antitumor activity and safety of this regimen in daily practice and especially in prolonged administration and to explore factors that may influence clinical outcomes, by means of collecting real-world data.

\section{Materials and Methods}

Consecutive patients with locally advanced, unresectable, or metastatic NENs who received at least one cycle of CAPTEM based on a specific dosing schedule [16] from June 2007 to February 2018 were identified in 3 European Neuroendocrine Tumor Society (ENETS) certified NEN referral centers (Centers of Excellence) from different countries (Greece $n=34$, Poland $n=35$ and Israel $n=10)$. Patients were included if they had biopsy proven NEN with measurable disease in either computed tomography (CT) or magnetic resonance imaging. Patients had progressive disease (PD) on radiological follow-up, based on response evaluation criteria in solid tumors (RECIST) criteria applied by each center's dedicated radiologists, while on previous treatments $(n=60)$ or were treatmentnaive, with uncontrolled functional syndrome $(n=3)$ or high-volume (bulky) disease $(n=16)$. This was defined as disseminated bilobar hepatic metastases involving $>50 \%$ of the hepatic parenchyma (type III according to Frilling et al. [29]), while multiple bone metastases were also present in half of these patients $(n=8)$, necessitating tumor load reduction. Decision to initiate CAPTEM was made by the dedicated specialized tumor board of each center.

Patient and tumor characteristics (histology, grading, and secretory status), history of prior treatments, and functional imaging properties acquired from fluoro-deoxyglucose positron emission tomography (FDG-PET) and somatostatin receptor imaging (SRI, including SPECT with somatostatin analogs labeled with ${ }^{99 \mathrm{~m}} \mathrm{Tc}$ (Tektrotyd) or ${ }^{111} \mathrm{In}$ (Octreoscan) and Ga ${ }^{68}$ DOTATOC/ DOTATATE PET/CT) were retrospectively collected. Histopathological diagnosis was locally performed by each center's dedicated histopathologist based on existing ENETS classification system and Standards of Care. In order to assess the prognostic impact of the proliferation index Ki67, all patients, irrespective of their tumor origin, were categorized into 4 groups according to its value at the time of CAPTEM initiation: Group 1 Ki67 <3\%, Group 2 Ki67 3-20\%, Group 3 Ki67 21-55\%, and Group 4 Ki67 >56\%. These specific cutoffs were employed based on the current WHO 2017 nomenclature as well as data from the NORDIC NEC study showing patients with $\mathrm{Ki}-67<55 \%$ to be less responsive to platinum-based chemotherapy $[30,31]$. MGMT assessment was not routinely performed.

The dosing schedule consisted of CAP at $750 \mathrm{mg} / \mathrm{m}^{2}$ twice daily on days 1-14 and TEM at $200 \mathrm{mg} / \mathrm{m}^{2}$ once daily on days $10-14$, in a 28-day cycle. When appropriate, doses were adjusted to minimize the number of tablets required or the possibility of toxicity in high-risk patients.

Patients were followed-up at 3-month intervals with imaging studies (CT or magnetic resonance imaging) to assess disease response to treatment and had a complete blood count, creatinine, and liver function tests obtained at several points during each chemotherapy cycle. Tumor responses were assessed based on (RECIST) version.1.1 [32] by each center's dedicated radiologist.
Stable disease (SD) and PR were considered clinically favorable responses, in contrast with $\mathrm{PD}$.

Duration of CAPTEM administration, reason to stop treatment, and side effects were extracted from patient files on May 2018 (time of study data collection). To avoid immortal time bias, baseline for overall survival (OS) and PFS analyses was defined as the first date on which patients started the CAPTEM regimen. The patients who died during CAPTEM treatment without evidence of PD were not included in response analysis. All patients were included in survival analysis. Patients still receiving CAPTEM without exhibiting progression entered the analysis with PFS equal to the duration of treatment. Side effects were graded using the National Cancer Institute Common Terminology Criteria for Adverse Events CTCAE version 5.0 [33].

Although CAPTEM represents a widely accepted therapy for NENs, each individual agent has not been registered for the specific indication and thus approval by local ethical and scientific committees as well as the national regulatory authorities and health-care providers was obtained on an individual basis for all patients included in the study. Signed informed consent for inclusion in the study was provided by the patients as required by the institutional review boards of the participating centers, which included the EKPA-Laiko ENETS Center of Excellence (Athens, Greece), the Silesian Medical University ENETS Center of Excellence (Katowice, Poland), and the Hadassah-Hebrew University Medical Center ENETS Center of Excellence (Jerusalem, Israel). The STROBE statement was followed to ensure quality of data reporting [34].

\section{Statistical Analysis}

All statistical analyses (frequencies, descriptive statistics, Kaplan-Meyer curves, log-rank tests, Cox regression) were done with the SPSS 23.0 software package (IBM SPSS Statistics, Armonk, NY, USA). Nonparametric data are presented as medians with range. Differences in baseline characteristics between patient groups were assessed using the chi-square test and the Fischer's exact test, as appropriate. PFS and OS curves were generated with the Kaplan-Meier method. Crude analysis of PFS and OS in different subgroups was stratified by clinicopathological parameters and computed with the Mantel-Cox log-rank test. This univariate analysis for PFS and OS was followed by adjusted Cox regression for multivariate analysis to identify predictors of survival. A $p$ value $<0.05$ was considered statistically significant and 95\% CIs are given for survival estimates. Receiver operating characteristic (ROC) curve analysis was used to estimate the predictive ability of Ki67 for favorable response to CAPTEM and identify a possible cutoff value for $\mathrm{Ki}-67$ index with regard to response rate (favorable response = $\mathrm{PR}+\mathrm{SD}$, no response $=\mathrm{PD})$.

\section{Results}

\section{Patient Characteristics}

Patient and tumor characteristics are summarized in Table 1 . The CAPTEM combination was administered in 79 NEN patients (31 women) at a median age of 60 years (range 30-84). Of the 79 patients included in the study, 74 harbored sporadic tumors and 5 familial NENs in the context of multiple endocrine neoplasia type 1 syndrome. 
Table 1. Baseline patient and tumor characteristics of the study cohort

\begin{tabular}{|c|c|c|}
\hline Characteristics & $n$ & $\%$ \\
\hline & 79 & 100 \\
\hline \multicolumn{3}{|l|}{ Gender } \\
\hline Male & 48 & 60.8 \\
\hline Female & 31 & 39.2 \\
\hline \multicolumn{3}{|l|}{ Age, years, median (range) } \\
\hline At diagnosis & \multicolumn{2}{|c|}{$55(18-81)$} \\
\hline At CAPTEM initiation & \multicolumn{2}{|c|}{$60(29-84)$} \\
\hline \multicolumn{3}{|l|}{ Inheritance } \\
\hline Sporadic & 74 & 93.7 \\
\hline Familial (MEN1) & 5 & 6.3 \\
\hline \multicolumn{3}{|l|}{ Primary tumor site } \\
\hline Pancreas & 30 & 38.0 \\
\hline GI-NEN (without pancreas) & 15 & 19.0 \\
\hline Lung/thymus & 17 & 21.5 \\
\hline UPO & 14 & 17.7 \\
\hline Various other & 3 & 3.8 \\
\hline \multicolumn{3}{|c|}{ Grading at CAPTEM initiation (GEP-NENs, UPO, various) } \\
\hline G1 $($ Ki67 <3\%) & 11 & 13.9 \\
\hline G2 (Ki67 3-20\%) & 27 & 34.2 \\
\hline G3 $($ Ki67 > 20\%) & 24 & 30.4 \\
\hline \multicolumn{3}{|l|}{ Grading at CAPTEM initiation (lung/thymus) } \\
\hline Typical carcinoid & 2 & 2.5 \\
\hline Atypical carcinoid & 11 & 13.9 \\
\hline Large-cell NEC & 4 & 5.1 \\
\hline \multicolumn{3}{|c|}{ Ki67 Groups at CAPTEM initiation (entire cohort) } \\
\hline Group $1(\mathrm{Ki} 67<3 \%)$ & 12 & 15.2 \\
\hline Group 2 (Ki67 3-20\%) & 37 & 46.8 \\
\hline Group 3 (Ki67 21-55\%) & 21 & 26.6 \\
\hline Group 4 (Ki67 > 56\%) & 9 & 11.4 \\
\hline \multicolumn{3}{|l|}{ Secretory status } \\
\hline Functioning & 25 & 31.6 \\
\hline Carcinoid syndrome & 11 & 13.9 \\
\hline Insulinoma & 4 & 5.1 \\
\hline Ectopic Cushing's & 4 & 5.1 \\
\hline Gastrinoma & 2 & 2.5 \\
\hline VIPoma & 2 & 2.5 \\
\hline PTHrP & 1 & 1.3 \\
\hline Ectopic calcitonin secretion & 1 & 1.3 \\
\hline Nonfunctioning & 54 & 68.4 \\
\hline \multicolumn{3}{|l|}{ Prior surgery } \\
\hline Yes & 39 & 49.4 \\
\hline No & 40 & 50.6 \\
\hline \multicolumn{3}{|l|}{ SRI } \\
\hline SRI positive & 57 & 72.2 \\
\hline SRI negative & 22 & 27.8 \\
\hline \multicolumn{3}{|c|}{ Combined functional imaging (SRI and FDG-PET) } \\
\hline SRI positive/PET positive & 23 & 29.1 \\
\hline SRI negative/PET positive & 11 & 13.9 \\
\hline SRI positive/PET negative & 4 & 5.1 \\
\hline \multicolumn{3}{|l|}{ Previous systemic treatments } \\
\hline No prior treatment & 19 & 24.1 \\
\hline Chemotherapy & 31 & 39.2 \\
\hline PRRT & 14 & 17.7 \\
\hline \multicolumn{3}{|l|}{ Molecular-targeted agents } \\
\hline (everolimus, sunitinib) & 23 & 29.1 \\
\hline \multicolumn{3}{|l|}{ CAPTEM line of treatment } \\
\hline 1st line (naïve) & 19 & 24.1 \\
\hline$\geq 2$ nd line (pretreated) & 60 & 75.9 \\
\hline \multicolumn{3}{|l|}{ Concomitant treatment } \\
\hline SSA & 53 & 67.1 \\
\hline Cytoreduction (ablation, embolization) & 9 & 11.4 \\
\hline
\end{tabular}

Based on primary tumor location, cases were grouped into GI-NENs $(n=15)$, including the stomach, the duodenum, the small intestine and the colon as primaries, panNENs $(n=30)$, Lung/Thymic NENs $(n=17)$, unknown primary origin neuroendocrine neoplasms (UPONENs, $n=14)$, and various NENs $(n=3)$ from rare primaries (nasopharynx, kidney, uterus).

Twenty-one patients had a functioning tumor at diagnosis, and 4 additional patients developed a secretory syndrome metachronously. Carcinoid syndrome was the most prevalent $(n=11)$ followed by insulinoma $(n=4)$ and ectopic Cushing's syndrome $(n=4)$, with other functioning syndromes represented less frequently.

All patients had SRI (Octreoscan, Tektrotyd, or $\mathrm{Ga}^{68}$ DOTATOC/DOTATATE PET) performed before CAPTEM initiation, showing pathological uptake in 57 cases. FDG-PET was performed in 38 patients, of which 34 had a positive scan.

Surgery with curative/debulking intent at/immediately after NEN diagnosis was performed in 39 patients, while in 40 patients the disease was considered unresectable at diagnosis and received systemic medical treatment instead. At the time of CAPTEM treatment initiation, all patients had metastatic or locally advanced (inoperable) disease (stage III-IV). CAPTEM was administered as a first-line medical treatment due to bulky disease $(n=16)$ or uncontrolled functional syndrome $(n=3)$ without evidence of prior progression (naive) and as subsequent lines after disease progression with other medical agents in 60 patients (pretreated). Somatostatin analogs were coadministered with CAPTEM in 53 patients, while in 9 patients cytoreductive procedures (radiofrequency ablation, chemoembolization) during the period of CAPTEM treatment were performed in order to control functional syndrome or reduce tumor load.

\section{Duration of Treatment}

CAPTEM was administered until disease progression, serious toxicity, death, or clinical considerations for longterm toxicity despite the favorable response. Median duration of treatment for the entire cohort was 12.1 months (range 0.6-55.6). The CAPTEM combination was administered in 42 patients until disease progression (median treatment duration 4.4 months, range $0.6-38.7$ ). Four patients died because of widespread disease and disease-related complications while receiving CAPTEM after a median of 2.1 months. Severe and intolerable side effects were the reason to stop treatment in 8 patients after a median period of 6.5 months (range 1.7-14.8). 
Table 2. Responses with CAPTEM chemotherapy regimen in different subgroups

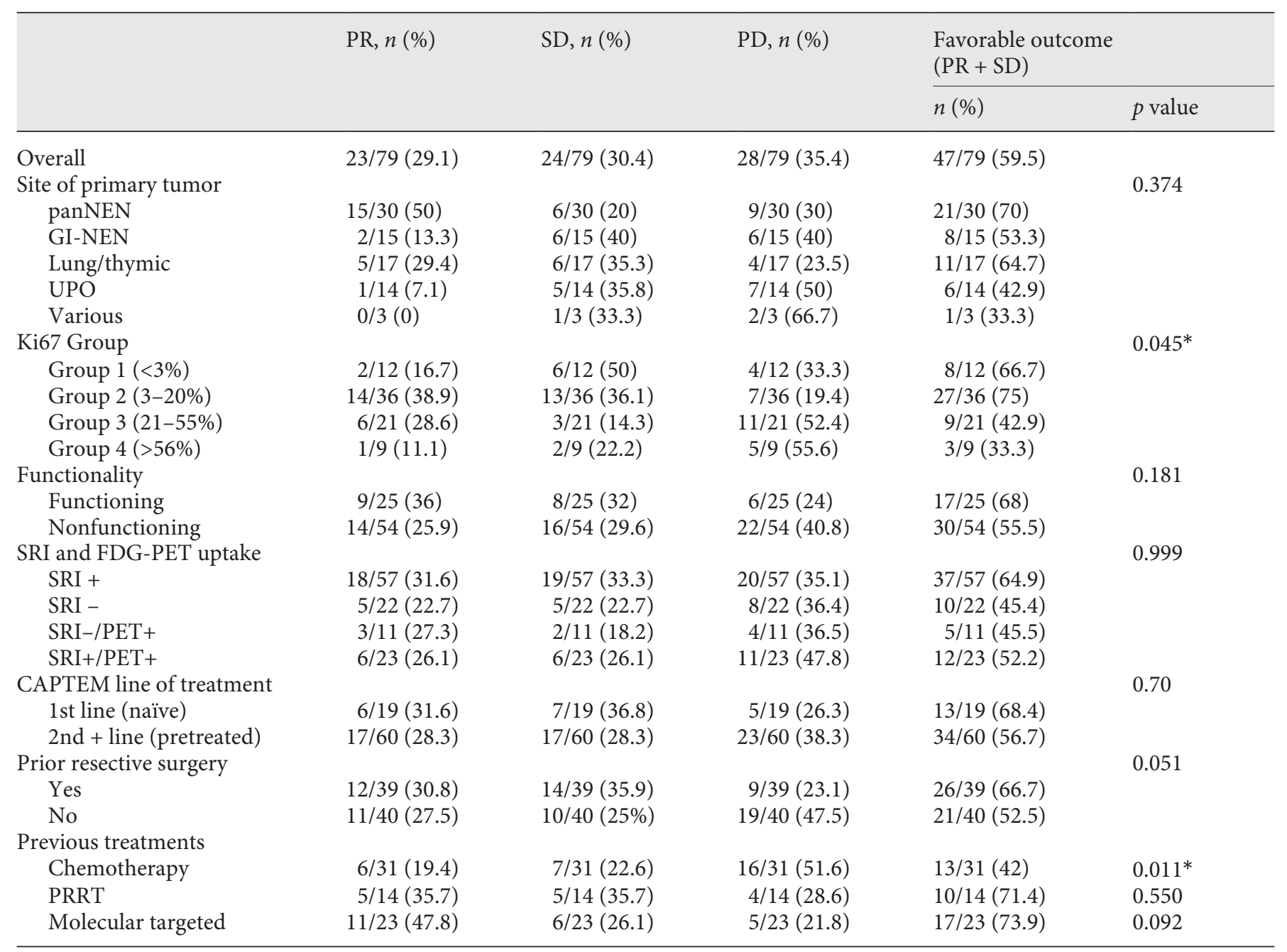

CAPTEM, capecitabine and temozolomide combination; PR, partial response; PD, progressive disease; panNEN, pancreatic neuroendocrine neoplasms; GI-NEN, gastrointestinal neuroendocrine neoplasm; UPO, UPO, unknown primary origin; FDG-PET, fluoro-deoxyglucose positron emission tomography; SRI, somatostatin receptor imaging; PRRT, peptide receptor radiation treatment. * indicates statistical significance at $p<0.05$.

A further 7 patients stopped treatment despite a favorable response ( 2 patients with $\mathrm{SD}, 5$ patients with $\mathrm{PR}$ ) based on clinical considerations of long-term toxicity. In this group, median duration of treatment was 25.8 months (10.1-45.4 months). Notably, of these 7 patients, only 2 have exhibited disease progression 15 and 18 months after CAPTEM discontinuation, while the rest remained stable after a follow-up of 3-12 months. Finally, 17 patients were still receiving CAPTEM with favorable response at the time of study data collection after a median period of 19.6 months (range 2.8-55.6 months).
Regarding duration of CAPTEM administration, 27 patients have received CAPTEM for $>12$ months (median 23.7 months, range 12.8-55.6). In this group of patients which mostly consisted of panNENs and lung/thymic NENs (a total of 24 out of 27), only 8 have exhibited PD (median PFS 17.7 months, range 12.8-38.7) while the remaining are showing favorable responses without significant toxicity. Given the lack of evidence-based recommendations on treatment duration, the decision to continue CAPTEM treatment cycles beyond 12 months was made on clinical judgement per case and independently of best-observed response, since $15 / 27$ patients had shown 
Table 3. Multivariate Cox-regression model for PFS and OS for all patients receiving the CAPTEM regimen from baseline (CAPTEM initiation)

\begin{tabular}{|c|c|c|c|c|c|c|}
\hline \multirow[t]{2}{*}{ Prognostic factor } & \multicolumn{3}{|l|}{ PFS } & \multicolumn{3}{|l|}{ OS } \\
\hline & HR & $95 \% \mathrm{CI}$ & $p$ value & $\mathrm{HR}$ & $95 \% \mathrm{CI}$ & $p$ value \\
\hline Gender & & & 0.423 & & & 0.526 \\
\hline Male & 1 & & & 1 & & \\
\hline Female & 0.77 & $0.4-1.5$ & & 1.3 & $0.6-3$ & \\
\hline Age at baseline & & & 0.13 & & & $0.024^{*}$ \\
\hline$<50$ & 1 & & & 1 & & \\
\hline $51-65$ & 1.1 & $0.5-2.3$ & 0.800 & 0.3 & $0.1-1$ & 0.045 \\
\hline$>66$ & 0.5 & $0.2-1.1$ & 0.081 & 0.3 & $0.1-0.8$ & 0.013 \\
\hline Site of primary & & & $0.002^{*}$ & & & $0.001^{*}$ \\
\hline Pancreas & 1 & & & 1 & & \\
\hline Gastrointestinal tract & 0.3 & $0.1-0.8$ & 0.009 & 0.2 & $0.1-0.7$ & 0.011 \\
\hline Lung/thymic & 1.7 & $0.6-4.3$ & 0.303 & 2.4 & $0.7-8.4$ & 0.65 \\
\hline UPO & 0.3 & $0.1-0.8$ & 0.018 & 0.2 & $0.1-0.9$ & 0.029 \\
\hline Ki67 groups & & & 0.146 & & & 0.639 \\
\hline Ki67 <3\% & 1 & & & 1 & & \\
\hline Ki67 3-20\% & 0.9 & $0.3-3.6$ & 0.936 & 0.7 & $0.1-3.9$ & 0.719 \\
\hline Ki67 21-55\% & 0.3 & $0.1-1.1$ & 0.078 & 0.5 & $0.1-2$ & 0.299 \\
\hline Ki67 $>56 \%$ & 0.5 & $0.2-1.5$ & 0.235 & 0.9 & $0.2-3.1$ & 0.838 \\
\hline Prior resective surgery & & & $0.021^{*}$ & & & $0.028^{*}$ \\
\hline No & 1 & & & 1 & & \\
\hline Yes & 2.4 & $1.1-4.9$ & & 3 & $1.1-8.1$ & \\
\hline Prior chemotherapy & & & & & & 0.208 \\
\hline No & 1 & & & 1 & & \\
\hline Yes & 0.8 & $0.4-1.8$ & 0.573 & 0.5 & $0.2-1.5$ & \\
\hline
\end{tabular}

PEF, progression-free survival; OS, overall survival; CAPTEM, capecitabine and temozolomide combination; UPO, unknown primary origin. ${ }^{*}$ indicates statistical significance at $p<0.05$.

SD only. It should also be noted that in 3 patients dosing schedules were modified (given every other month) after the first year of CAPTEM treatment.

\section{Activity}

Best response during CAPTEM administration was documented according to RECIST criteria at any time after treatment initiation. Overall, 23 patients (29.1\%) exhibited PR, 24 patients showed SD (30.4\%), 28 patients had radiological PD (35.4\%), and 4 patients died during treatment without evidence of PD (Table 2). No case with complete response to CAPTEM (complete disappearance of tumor) was observed in our cohort, although 1 patient showed considerable response allowing the surgical removal of his previously unresectable localized panNEN. Age, gender, and functionality were not found to be associated with favorable responses.

Favorable responses $(\mathrm{PR}+\mathrm{SD})$, although not statistically significant between different primary tumor loca- tions, were more common in patients with panNENs and lung/thymic NENs reaching 70 and $64.7 \%$, respectively. Patients with GI-NENs and UPO-NENs also responded favorably, in 53.3 and $42.9 \%$ of the cases, respectively. The highest PR rate was observed in patients with panNENs (50\%).

After stratifying patients according to Ki67 LI in the aforementioned groups, favorable response rates were better for groups 1 and 2 (66.7 and 75\%, respectively) compared to the higher Ki67 groups (group 3: $42.9 \%$ and group 4: $33.3 \%$, respectively; $p$ value $=0.045)$. PRs were more common in Group 2 (38.9\%) compared to Group 1 (16.7\%) in which the majority of patients (50\%) exhibited disease stability as a favorable outcome. However, Ki-67 in ROC analysis was a poor predictor for treatment response (area under the curve $=0.678$ ) and was not associated with PFS on multivariate analysis (Table 3 ).

Functional imaging properties in SRI and FDG-PET, alone or in combination, were not associated with best- 
recorded response to CAPTEM. Patients who had undergone prior resective or cytoreductive surgery demonstrated a trend toward better favorable response compared with those who received medical therapy only $(p$ value $=0.051$ ).

Regarding other medical treatments, favorable responses with CAPTEM were not different between naive and pretreated patients and were not associated with concurrent somatostatin analog use. Additionally, among previously administered medical treatments, only prior chemotherapy was associated with worse favorable responses $(p$ value $=0.011)$.

\section{Survival Analysis of Clinicopathological Prognostic}

Factors at Baseline

At the time of study data collection, 36 patients had died and 43 were still alive after a median follow-up of 21.8 months (range 2.8-63.5). Median PFS and OS from baseline for the entire cohort were 10.1 months (6-14.2) and 102.9 months (43.3-162.5), respectively (Fig. 1a, b). PFS and OS, as well as univariate and multivariate survival analyses regarding age, gender, primary tumor site, Ki67 LI, functional status, functional imaging, prior surgery, and concomitant or prior medical treatments are presented in Tables 3 and 4. In crude analysis, low Ki67 LI values (groups 1 and 2) and chemotherapy-naive patients were associated with favorable response rates $(p=$ 0.045 and 0.011 , respectively). On univariate analysis, PFS and OS were significantly longer for panNENs and lung/thymic NENs (log-rank $p<0.0001$ and $<0.0001$ ), Ki67 group 2 (log-rank $p<0.0001$ and 0.005$)$, and patients not pretreated with chemotherapy $(\log$-rank $p<$ 0.002 and 0.001) (Table 4; Fig. 1c, d). On multivariable analysis, only the primary tumor site (pancreatic and lung/thymic NENs) and prior resective or cytoreductive surgery were independent prognostic factors for PFS $(p=$ 0.002 and 0.021 , respectively) and OS ( $p=0.001$ and 0.028 , respectively); age at baseline was significant for OS $(p=0.024)$ with patients older than 50 years old being in higher risk (Table 3 ).

\section{Side Effects}

The CAPTEM combination treatment was generally well tolerated. From the entire cohort of 79 patients, 38 (48.1\%) experienced any side effect, mostly grade 1 and 2 toxicities. Table 5 summarizes the most common side effects and the frequency of their appearance. Fatigue, GI symptoms (nausea, vomiting, diarrhea, abdominal pain), and hematological complications (reduced platelet, hematocrit, and white blood cell count) were the most fre- quent grades 1-2 side effects noticed. Only 4 patients developed serious (grades 3-4) toxicities mostly regarding bone marrow suppression and had to discontinue treatment: 2 patients presented with grade 3 thrombocytopenia, 1 patient with grade 3 anemia and 1 patient with grade 4 pancytopenia. Another 4 patients stopped medication with mild side effects after denial of further treatment continuation (1 with aspirin-related gastric bleeding and 3 with fatigue). Seven patients discontinued treatment in the first 9 months post treatment initiation and 1 patient, with gastric bleeding, stopped after 15 months of treatment (median 6.5 months, range 1.7-14.8). All other patients who developed grades 1-2 side effects were given supportive symptomatic medication and/or slightly reduced CAPTEM dosages depending on clinical judgement and patient characteristics.

Four deaths occurred in patients receiving CAPTEM due to clinically documented disease progression (1 patient with large cell neuroendocrine carcinomas and brain metastases; 1 patient with aggressive and rapidly progressing gastric NEN G3; 1 patient with atypical lung NEN with bone metastases, carcinoid syndrome, and heart comorbidities; 1 patient with UPO-NEN G2 and uncontrolled carcinoid syndrome).

\section{Discussion}

The present study evaluates the activity and safety of CAPTEM regimen in a large number of NENs of various primaries and Ki67 values. The relatively large number of patients included as well as the use of the same dosing schedule by the 3 participating centers adds up to the strength of the current study.

Our study is also the first one to report a considerable number of patients $(n=27)$ who were treated with the CAPTEM combination for $>12$ months (median 23.7 months, range 12.8-55.6). The duration of treatment in patients who acquire long-term favorable responses to CAPTEM combination is a question that has not been answered yet with convincing data. As TEM is an alkylating agent that damages the DNA, development of secondary malignancies is a potential cause of concern that is partially supported by reports from TEM-treated glioblastoma patients presenting with myelodysplastic syndrome, leukemia or aplastic anemia [35-37]. No such reports exist for NEN patients despite available long-term follow-up data. Administration for up to 39 months has been reported by some studies $[16,22,26]$ without evidence of chronic myelotoxicity. In our effort to illustrate real-world practice we 

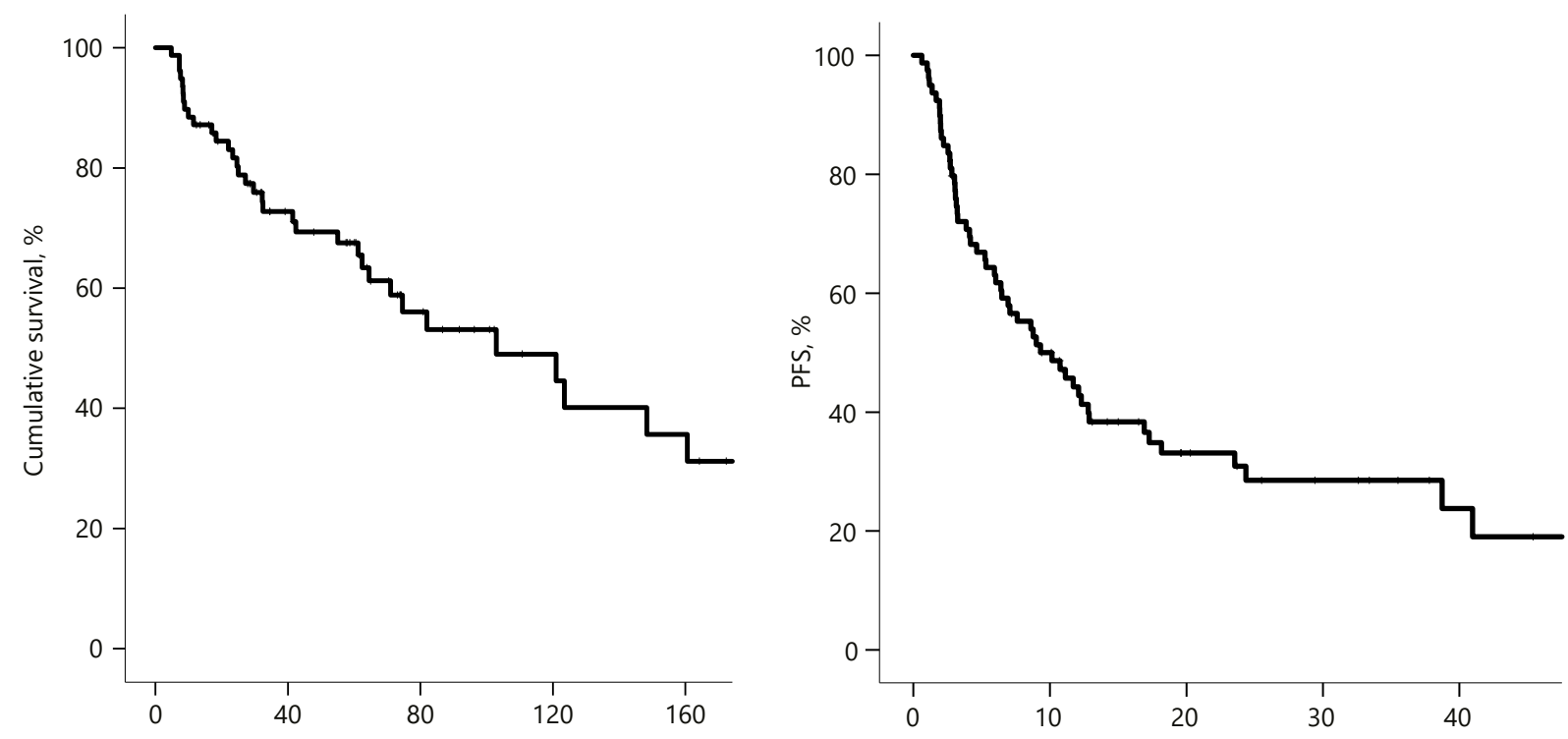

a

Number at risk 79
Time from diagnosis, months

43

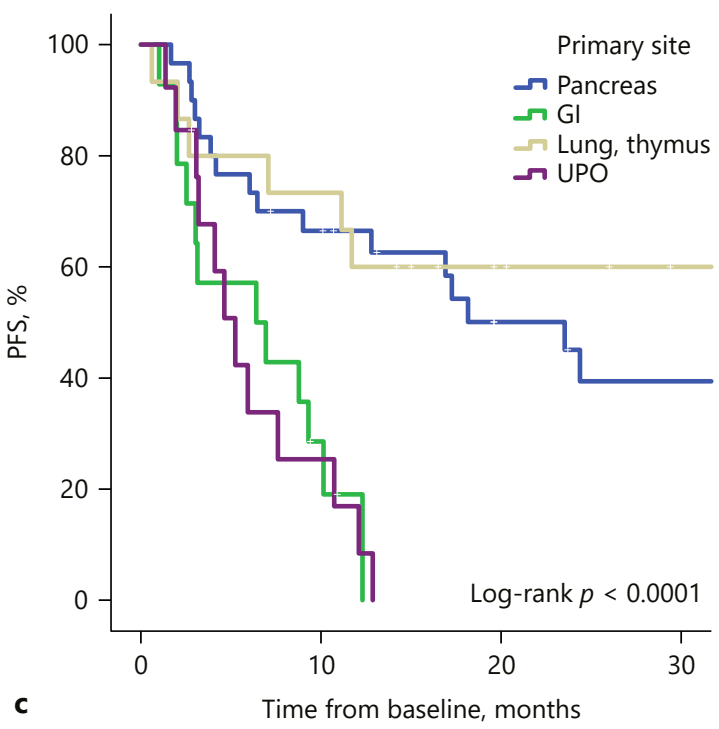

Primary

panNEN

GI-NEN

U N 17

UPO-NEN

\begin{tabular}{cc}
\multicolumn{2}{c}{ No. at risk } \\
19 & 10 \\
3 & 0 \\
11 & 5 \\
3 & 0
\end{tabular}

b

Time from treatment initiation, months

$\begin{array}{llllll}8 & \text { Number at risk } & 79 & 37 & 16 & 10\end{array}$

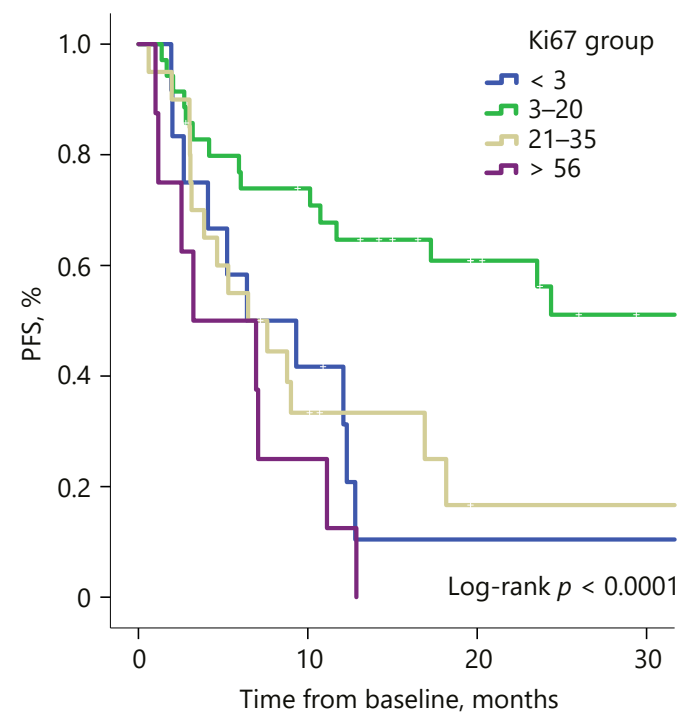

Ki67 value

No. at risk

$\begin{array}{lcccc}\text { Ki67 }<3 & 12 & 5 & 1 & 0 \\ \text { Ki67 3-20 } & 37 & 24 & 14 & 8 \\ \text { Ki67 } 21-55 & 21 & 6 & 1 & 1 \\ \text { Ki67 }>56 & 9 & 2 & 0 & 0\end{array}$


Table 4. PFS, OS rates, and crude analysis of PFS and OS according to the Mantel-Cox regression model for patient characteristics at baseline (CAPTEM initiation)

\begin{tabular}{|c|c|c|c|c|}
\hline Patient characteristics & $\begin{array}{l}\text { Median PFS ( } 95 \% \\
\text { CI), months }\end{array}$ & $\begin{array}{l}\text { Log-rank } \\
p \text { value }\end{array}$ & $\begin{array}{l}\text { Median OS } \\
(95 \% \mathrm{CI}), \text { months }\end{array}$ & $\begin{array}{l}\text { Log-rank } \\
p \text { value }\end{array}$ \\
\hline Site of primary & & $<0.0001^{*}$ & & $<0.0001^{*}$ \\
\hline Pancreas & $23.5(15.9-31.2)$ & & $83.9(-)$ & \\
\hline GI-NEN & $6.4(1.5-11.3)$ & & $13.4(8.4-18.4)$ & \\
\hline Lung/thymic & $\mathrm{NR}(-)$ & & $\mathrm{NR}(-)$ & \\
\hline UPO & $4.6(2.7-6.6)$ & & $17.3(0-45.2)$ & \\
\hline Ki67 Groups & & $<0.0001^{*}$ & & $0.005^{*}$ \\
\hline Ki67<3\% & $6.4(0-13.3)$ & & $28.2(0.6-55.8)$ & \\
\hline Ki67 3-20\% & $24.4(1.8-47)$ & & $83.9(0-169.7)$ & \\
\hline Ki67 21-55\% & $6.5(2.2-10.7)$ & & $14.4(2.7-26.1)$ & \\
\hline Ki67 $>56 \%$ & $3.2(1.2-5.3)$ & & $17.3(4.2-30.4)$ & \\
\hline Functional imaging - SRI status & & 0.067 & & 0.073 \\
\hline Negative & $6.9(0.7-13.2)$ & & $17.9(7.2-28.6)$ & \\
\hline Positive & $12.1(7.6-16.6)$ & & $55.8(4.5-107.1)$ & \\
\hline Functional status & & 0.176 & & 0.667 \\
\hline No & $6.9(1.3-12.6)$ & & $33.6(16.4-50.8)$ & \\
\hline Yes & $16.9(5.6-28.2)$ & & $83.9(12.3-155.5)$ & \\
\hline Prior resective surgery & & 0.169 & & 0.491 \\
\hline No & $5.9(0.1-11.8)$ & & $33.6(15.3-51.9)$ & \\
\hline Yes & $12.1(7.6-16.6)$ & & $\mathrm{NR}(-)$ & \\
\hline Concurrent SSA use & & 0.053 & & 0.093 \\
\hline Yes & $5.3(0.5-10.1)$ & & $22(5-39)$ & \\
\hline No & $12.3(8.9-15.7)$ & & $55.8(20.7-90.9)$ & \\
\hline Prior PRRT & & 0.777 & & 0.784 \\
\hline No & $9(4.5-13.5)$ & & $55.8(9.9-101.7)$ & \\
\hline Yes & $10.7(5.7-15.8)$ & & $28.2(10.4-46)$ & \\
\hline Prior MTT & & 0.144 & & 0.485 \\
\hline No & $6.5(4-8.9)$ & & $55.8(9.9-101.7)$ & \\
\hline Yes & $16.9(10.3-23.5)$ & & $\mathrm{NR}(-)$ & \\
\hline Prior chemotherapy & & $0.002^{*}$ & & $0.001^{*}$ \\
\hline No & $12.8(0-26.4)$ & & NR & \\
\hline Yes & $6.5(2.7-10.2)$ & & $17.3(11.8-22.8)$ & \\
\hline Order of CAPTEM regimen & & 0.372 & & 0.354 \\
\hline 1 st & $24.4(1.8-46.9)$ & & NR & \\
\hline $2 \mathrm{nd}$ & $7.1(3.2-10.9)$ & & $33.6(4.4-62.8)$ & \\
\hline $3 \mathrm{rd}$ & $10.7(6.6-14.9)$ & & $22.7(9.3-36.1)$ & \\
\hline 4 rth + & $11.1(1.1-21.2)$ & & $22(12.6-31.4)$ & \\
\hline
\end{tabular}

PEF, progression-free survival; OS, overall survival; CAPTEM, capecitabine and temozolomide combination; GI-NEN, gastrointestinal neuroendocrine neoplasm; UPO, unknown primary origin; SRI, somatostatin receptor imaging; SSA, somatostatin analogues; PRRT, peptide receptor radionuclide therapy; MTT, molecular-targeted therapies (i.e., everolimus, sunitinib). ${ }^{*}$ indicates statistical significance at $p<0.05$.

found one patient from our series to have reached 55 months of CAPTEM treatment, while 27 out of the 79 patients have received the combination for $>12$ months without any serious sequelae. Since no evidence-based recommendations exist on the optimal duration of therapy, decision to continue therapeutic cycles beyond currently available experience was made individually, based on pa- tients' preference and/or to avoid exacerbation of difficult to control functional syndromes. We also observed an alternative approach of reducing the frequency of therapeutic cycles after the first year of administration (from monthly to every other month) in a few patients with favorable responses. This intermittent drug administration strategy was followed in 3 patients in order to minimize toxicity; 
Table 5. Type, frequency, and severity (grade) of adverse effects noticed in patients treated with CAPTEM, according to CTCAE version 5.0

\begin{tabular}{|c|c|c|c|c|c|c|c|c|}
\hline \multirow[t]{3}{*}{ Toxicity } & \multicolumn{8}{|c|}{ Maximum toxicity grade } \\
\hline & \multicolumn{2}{|l|}{1} & \multicolumn{2}{|l|}{2} & \multicolumn{2}{|l|}{3} & \multicolumn{2}{|l|}{4} \\
\hline & $n$ & $\%$ & $n$ & $\%$ & $n$ & $\%$ & $n$ & $\%$ \\
\hline \multicolumn{9}{|l|}{ Hematologic } \\
\hline Anemia & 8 & 10.1 & 1 & 1.3 & 1 & 1.3 & 1 & 1.3 \\
\hline Neutropenia & 5 & 6.3 & 1 & 1.3 & - & & 1 & 1.3 \\
\hline Thrombocytopenia & 5 & 6.3 & 2 & 2.5 & 2 & 2.5 & 1 & 1.3 \\
\hline \multicolumn{9}{|l|}{ Gastrointestinal } \\
\hline Abdominal pain & 6 & 7.6 & 5 & 6.3 & - & - & - & - \\
\hline Nausea & 6 & 7.6 & 2 & 2.5 & - & - & - & - \\
\hline Constipation & - & & 2 & 2.5 & - & - & - & - \\
\hline Diarrhea & 4 & 5.1 & 4 & 5.1 & - & - & - & - \\
\hline Anorexia & 2 & 2.5 & 2 & 2.5 & - & - & - & - \\
\hline GI-bleeding & - & & 1 & 1.3 & - & - & - & - \\
\hline \multicolumn{9}{|l|}{ Hepatotoxicity } \\
\hline Hepatobiliary disorders & 2 & 2.5 & 1 & 1.3 & - & - & - & - \\
\hline \multicolumn{9}{|l|}{ Nephrotoxicity } \\
\hline Chronic kidney disease & 1 & 1.3 & 3 & 3.8 & - & - & - & - \\
\hline \multicolumn{9}{|l|}{ Mucocutaneous } \\
\hline Stomatitis & 8 & 10.1 & - & - & - & - & - & - \\
\hline Palmar-plantar syndrome & 3 & 3.8 & - & - & - & - & - & - \\
\hline \multicolumn{9}{|l|}{ Other } \\
\hline Fatigue & 12 & 15.2 & 6 & 7.6 & - & - & - & - \\
\hline Peripheral sensory neuropathy & 2 & 2.5 & - & & - & - & - & - \\
\hline
\end{tabular}

CAPTEM, capecitabine and temozolomide combination; GI, gastrointestinal.

however, it needs further clinical testing. It is also encouraging that in the 7 cases that treatment was discontinued due to long-term toxicity concerns, only 2 patients had PD after a considerable PFS of $>12$ months. In such cases, CAPTEM rechallenge could arise as a potential option but needs to be further studied for its efficacy and safety.

As far as activity is concerned, although the highly heterogeneous patient population precludes definite conclusions, an overall favorable response $(\mathrm{PR}+\mathrm{SD})$ was observed in $59.5 \%$ of the entire cohort. This result is lower than the $70-80 \%$ rate reported $[16,22,23,26,38,39]$, but this could be attributed to the inclusion of a significant number of non-panNENs as well as tumors with high Ki67 LI in our cohort. Patients with panNENs and lung/ thymic NENs seem to benefit more in absolute rates compared with GI-NENs and UPO NENs, although this was not found to be statistically significant. Indeed, panNEN patients from our cohort had a favorable response rate of $70 \%$ in agreement with previous studies and exhibited the highest PR rate of 50\%. It should however be noted that favorable response rates for GI-NENs and UPO NENs, although lower compared to their pancreatic and thoracic counterpart, were demonstrated in almost half the patients treated with the CAPTEM combination.

PFS for the entire cohort was 10.1 months; however, differences between subgroups based on primary site of origin were considerable. Median PFS for panNEN patients in our cohort was 23.5 months (range 15.9-31.2), which is significantly longer than the median of 18 and 16.2 months reported in a previous [16] and a recent multicenter study [39]. Lung/thymic NENs did not reach median PFS in our analysis, while GI-NENs and UPO NENs exhibited low median PFS rates (3-6 months). Importantly, PFS benefit was evident in patients with panNENs and lung/thymic NENs at CAPTEM treatment in both crude and multivariable Cox-regression analyses. These significant differences have been reported by other researchers and attributed to the lower MGMT expression in panNENs compared with other primaries, possibly allowing TEM to be more effective [18].

Concerning the potential role of Ki67 LI as a predictor of outcome, in our series, lower Ki67 LI tumors (Group 1 
and Group 2) appeared to be associated with higher favorable responses compared to Group 3 and 4 (66.7 and 75\% vs. 42.9 and $33.3 \%$ ). It should be noted though that in Group 1 SD was the most common response (50\% of the entire group), rather than tumor shrinkage (16.7\%). On the other hand, patients with Ki67 LI 3-20\% (Group 2) exhibited mainly PR. They also seemed to benefit more in terms of PFS (Fig. 1d), as these patients obtained a PFS of 24.4 months compared with patients in Groups 3 and 4 who had a PFS of 6.5 and 3.2 months, respectively, but also with Group 1 patients (6.4 months). This unexpectedly short PFS for Ki67 Group 1 patients compared with Group 2 is probably attributed to the different distribution of primary tumors in these patient groups. Group 1 was mostly GIand UPO-NENs (9/12), whereas Group 2 included more lung/thymic and panNENs (29/37) without any small or large intestinal NEN. In addition, lung/thymic and panNENs both in the literature $[14,18,27]$, and in our study, exhibit better favorable response rates and longer PFS with CAPTEM. This is probably the reason why multivariate analysis failed to detect any predictive value of Ki67 for PFS and favorable responses and ROC analysis indicated poor prognostic value for favorable response (Fig. 2).

In the literature, similar results have also been reported on the predictive role of Ki67 even in patients with the same primary tumor. In a study with 61 panNENs, 28 tumors with Ki-67 $>5 \%$ showed an ORR (CR + PR) of 64 vs. $29 \%$ for the 31 panNENs with Ki-67 $<5 \%(p=0.006)$ [40]. Likewise, in another study of various NENs, an ORR of $29 \%$ in tumors with Ki-67 $<2$ and 39\% in NENs with Ki$672-20 \%$ was reported [24]. In pretreated mixed cases of panNENs and non-panNENs, PFS was not associated with $\mathrm{Ki}-67 \mathrm{LI}$ in 2 recent studies [21, 22]. From this evidence, it appears that CAPTEM is less effective in low Ki67 tumors $(<2-5 \%)$, but this needs further clarification in appropriately designed trials.

We also observed a trend for better favorable responses for patients who had undergone prior resective/cytoreductive surgery at NEN diagnosis. However, this finding could be the result of selection bias as patients with less contraindications for surgery or with less extensive and aggressive disease were treated surgically. Similarly, selection bias could explain why prior chemotherapy seemed to be associated with worse PFS and OS rates in our study.

The CAPTEM regimen in our study was well tolerated with a toxicity profile similar to the one reported by other researchers [22, 39, 41]. However, the retrospective collection of data and the lack of quantifiable information with appropriate questionnaires on patients' symptoms while on chemotherapy, limits our ability to determine how this

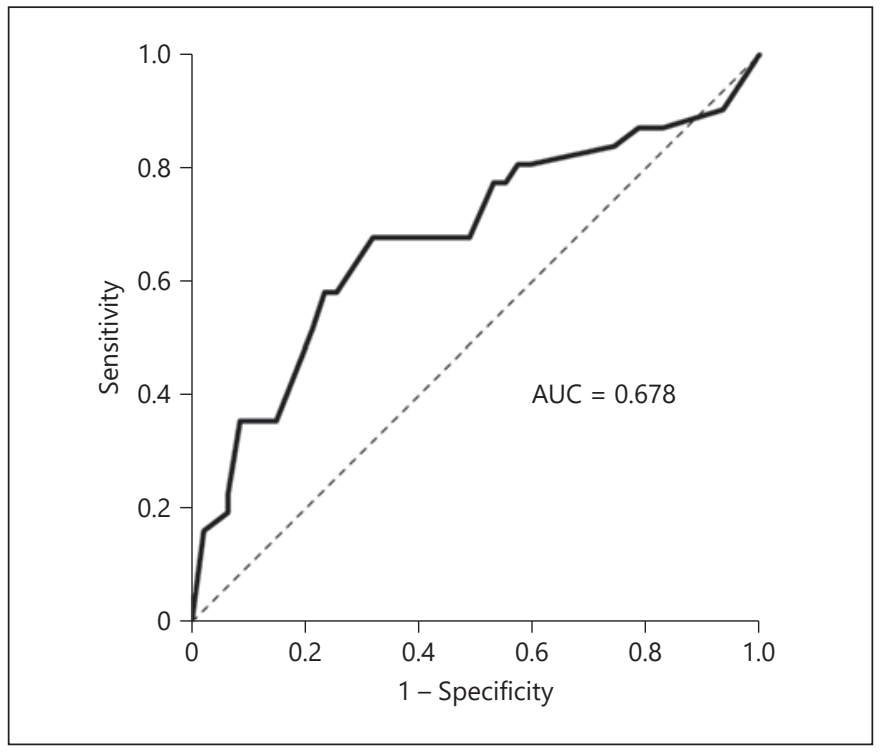

Fig. 2. ROC analysis of Ki-67 with regard to favorable responses $(\mathrm{PR}+\mathrm{SD})$ to CAPTEM regimen. AUC, area under the curve.

regimen impacted patients' quality of life. Another interesting observation is that almost all serious side effects necessitating treatment discontinuation occurred during the first 9 months of CAPTEM administration.

Limitations of our study include all inherent limitations of observational retrospective analyses, especially the potential selection bias toward long survivors and the heterogeneous population of patients without wellmatched subgroups and control groups. Lack of centralized radiological and histological assessment also poses a potential limitation of our study. However, it should be noted that histological diagnosis and radiological assessment were verified in each ENETS-certified center by dedicated specialists according to ENETS Guidelines and Standards of Care. Many of our results are in agreement with similar published studies; however, there are differences in certain areas that are also debatable and unclear in the literature, such as the role of Ki67, functional status, the imaging properties in FDG-PET/CT and SRI, and the effectiveness of this regimen in heavily pretreated patients. Although we studied a population of 79 patients who all received CAPTEM with the same dosing schedule, the heterogeneity of the entire cohort and the small sample size in certain subgroups may account for the lack of statistical significance observed for several variables. Our series is a real-world study of retrospective nature not designed and powered to address these questions, and further prospective trials are warranted. All data on OS 
should also be evaluated with caution, since due to the characteristic long-life expectancy of patients with NENs, various therapies are usually employed throughout the course of the disease that can affect OS.

Another potential weakness of this study is the lack of MGMT expression assessment. Due to the retrospective, real-world-data study design, it was not feasible to include an investigational marker such as MGMT, with limited -at the time-institutional and commercial availability. MGMT expression has been investigated as a prognostic factor of response to CAPTEM therapy in many tumor types. Despite the initial enthusiasm derived from a strong pathophysiological rationale and data from glioblastoma patients who responded significantly better to TEM when MGMT deficiency was documented in IHC, similar data in NEN patients using IHC or MGMT promoter methylation status have not been consistent in the literature [20,21, 40, 4244]. Recently, a large retrospective study of 144 CAPTEMtreated NEN patients found that MGMT deficiency (tested with IHC) could not predict response to treatment [21], a result cautioning against the use of MGMT expression as the sole predictor of response to CAPTEM and further reinforcing the need for better biomarkers to aid in clinical decision-making. Although MGMT expression was not included in our study, the responses observed were similar to those obtained from studies that included MGMT expression suggesting that the findings of the end points obtained were clinically meaningful and not directly affected by the absence of MGMT profiling.

\section{Conclusion}

In summary, this is one of the largest reported series of CAPTEM-treated NEN patients using the same dosing schedule in 3 specialized tertiary care centers from different countries. The results of our study add up to previous retrospective studies, suggesting that CAPTEM can be a suitable treatment option with limited toxicity and significant response rates for patients with various metastatic/unresectable NENs. The activity and safety of CAPTEM in a significant number of patients who were treated for $>12$ months (median 23.7 months, range 12.8-55.6) is also documented for the first time and important clinical questions are raised regarding treatment duration, intermittent administration, and safety concerns. There is a clear need for powered prospective trials that should address so far unresolved issues such as the predictive and prognostic impact of Ki67 LI, MGMT deficiency, and other molecular biomarkers, as well as the optimal sequence, duration and timing of the CAPTEM regimen in relation to the other approved targeted therapies.

\section{Disclosure Statement}

The authors have no conflicts of interest to declare.

\section{References}

1 Dasari A, Shen C, Halperin D, Zhao B, Zhou $\mathrm{S}, \mathrm{Xu} \mathrm{Y}$, et al. Trends in the Incidence, Prevalence, and Survival Outcomes in Patients With Neuroendocrine Tumors in the United States. JAMA Oncol. 2017 Oct;3(10):1335-42.

2 Kaltsas GA, Besser GM, Grossman AB. The diagnosis and medical management of advanced neuroendocrine tumors. Endocr Rev. 2004 Jun;25(3):458-511.

3 Bosman FT, Carneiro F, Hruban RH, Theise ND. WHO Classification of Tumours of the Digestive System. Fourth Edition. In: International Agency for Research on Cancer. 2010.

4 Lloyd RV, Osamura YR, Kloppel G, Rosai J. WHO classification of tumours of endocrine organs. In: WHO Press. 2017.

5 Travis WD, Brambilla E, Nicholson AG, Yatabe Y, Austin JH, Beasley MB, et al.; WHO Panel. The 2015 World Health Organization Classification of Lung Tumors: Impact of Genetic, Clinical and Radiologic Advances Since the 2004 Classification. J Thorac Oncol. 2015 Sep;10(9):1243-60.
6 Rinke A, Müller HH, Schade-Brittinger C, Klose KJ, Barth P, Wied M, et al.; PROMID Study Group. Placebo-controlled, doubleblind, prospective, randomized study on the effect of octreotide LAR in the control of tumor growth in patients with metastatic neuroendocrine midgut tumors: a report from the PROMID Study Group. J Clin Oncol. 2009 Oct;27(28):4656-63.

7 Caplin ME, Pavel M, Ćwikła JB, Phan AT, Raderer M, Sedláčková E, et al.; CLARINET Investigators. Lanreotide in metastatic enteropancreatic neuroendocrine tumors. N Engl J Med. 2014 Jul;371(3):224-33.

8 Yao JC, Fazio N, Singh S, Buzzoni R, Carnaghi C, Wolin E, et al.; RAD001 in Advanced Neuroendocrine Tumours, Fourth Trial (RADIANT-4) Study Group. Everolimus for the treatment of advanced, non-functional neuroendocrine tumours of the lung or gastrointestinal tract (RADIANT-4): a randomised, placebo-controlled, phase 3 study. Lancet. 2016 Mar;387(10022):968-77.
9 Yao JC, Shah MH, Ito T, Bohas CL, Wolin EM, Van Cutsem E, et al.; RAD001 in Advanced Neuroendocrine Tumors, Third Trial (RADIANT-3) Study Group. Everolimus for advanced pancreatic neuroendocrine tumors. N Engl J Med. 2011 Feb;364(6): 514-23.

10 Raymond E, Dahan L, Raoul JL, Bang YJ, Borbath I, Lombard-Bohas C, et al. Sunitinib malate for the treatment of pancreatic neuroendocrine tumors. N Engl J Med. 2011 Feb; 364(6):501-13.

11 de Mestier L, Dromain C, d'Assignies G, Scoazec JY, Lassau N, Lebtahi R, et al. Evaluating digestive neuroendocrine tumor progression and therapeutic responses in the era of targeted therapies: state of the art. Endocr Relat Cancer. 2014 Apr;21(3):R10520.

12 Basu B, Sirohi B, Corrie P. Systemic therapy for neuroendocrine tumours of gastroenteropancreatic origin. Endocr Relat Cancer. 2010 Jan;17(1):R75-90. 
13 Newlands ES, Stevens MF, Wedge SR, Wheelhouse RT, Brock C. Temozolomide: a review of its discovery, chemical properties, pre-clinical development and clinical trials. Cancer Treat Rev. 1997 Jan;23(1):35-61.

14 Chan JA, Stuart K, Earle CC, Clark JW, Bhargava P, Miksad R, et al. Prospective study of bevacizumab plus temozolomide in patients with advanced neuroendocrine tumors. J Clin Oncol. 2012 Aug;30(24):2963-8.

15 Ekeblad S, Sundin A, Janson ET, Welin S, Granberg D, Kindmark H, et al. Temozolomide as monotherapy is effective in treatment of advanced malignant neuroendocrine tumors. Clin Cancer Res. 2007 May;13(10): 2986-91.

16 Strosberg JR, Fine RL, Choi J, Nasir A, Coppola D, Chen DT, et al. First-line chemotherapy with capecitabine and temozolomide in patients with metastatic pancreatic endocrine carcinomas. Cancer. 2011 Jan;117(2):268-75.

17 Koumarianou A, Kaltsas G, Kulke MH, Oberg K, Strosberg JR, Spada F, et al. Temozolomide in Advanced Neuroendocrine Neoplasms: Pharmacological and Clinical Aspects. Neuroendocrinology. 2015;101(4):274-88.

18 Kulke MH, Hornick JL, Frauenhoffer C, Hooshmand S, Ryan DP, Enzinger PC, et al. O6-methylguanine DNA methyltransferase deficiency and response to temozolomidebased therapy in patients with neuroendocrine tumors. Clin Cancer Res. 2009 Jan; 15(1):338-45.

19 Murakami J, Lee YJ, Kokeguchi S, Tsujigiwa H, Asaumi J, Nagatsuka H, et al. Depletion of O6-methylguanine-DNA methyltransferase by O6-benzylguanine enhances 5 -FU cytotoxicity in colon and oral cancer cell lines. Oncol Rep. 2007 Jun;17(6):1461-7.

20 Walter T, van Brakel B, Vercherat C, Hervieu V, Forestier J, Chayvialle JA, et al. O6-Methylguanine-DNA methyltransferase status in neuroendocrine tumours: prognostic relevance and association with response to alkylating agents. Br J Cancer. 2015 Feb;112(3): 523-31.

21 Cives M, Ghayouri M, Morse B, Brelsford M, Black M, Rizzo A, et al. Analysis of potential response predictors to capecitabine/temozolomide in metastatic pancreatic neuroendocrine tumors. Endocr Relat Cancer. 2016 Sep;23(9):759-67.

22 Peixoto RD, Noonan KL, Pavlovich P, Kennecke HF, Lim HJ. Outcomes of patients treated with capecitabine and temozolamide for advanced pancreatic neuroendocrine tumors (PNETs) and non-PNETs. J Gastrointest Oncol. 2014 Aug;5(4):247-52.

23 Abbasi S, Kashashna A, Albaba H. Efficacy of capecitabine and temozolomide combination in well-differentiated neuroendocrine tumors: jordan experience. Pancreas. 2014 Nov; 43(8):1303-5.
24 Spada F, Antonuzzo L, Marconcini R, Gelsomino F, Fumagalli C, Messerini L, et al. Chemotherapy with capecitabine plus temozolomide (CAP-TEM) in patients with advanced neuroendocrine neoplasms (NENs): an Italian multicenter retrospective analysis. J Clin Oncol. 2015;33(15_suppl):e15174.

25 Ramirez RA, Beyer DT, Chauhan A, Boudreaux JP, Wang YZ, Woltering EA. The Role of Capecitabine/Temozolomide in Metastatic Neuroendocrine Tumors. Oncologist. 2016 Jun;21(6):671-5.

26 Fine RL, Gulati AP, Krantz BA, Moss RA, Schreibman S, Tsushima DA, et al Capecitabine and temozolomide (CAPTEM) for metastatic, well-differentiated neuroendocrine cancers: The Pancreas Center at Columbia University experience. Cancer Chemother Pharmacol. 2013 Mar;71(3):663-70.

27 Kulke MH, Stuart K, Enzinger PC, Ryan DP, Clark JW, Muzikansky A, et al. Phase II study of temozolomide and thalidomide in patients with metastatic neuroendocrine tumors. J Clin Oncol. 2006 Jan;24(3):401-6.

28 Kotteas EA, Syrigos KN, Saif MW. Profile of capecitabine/temozolomide combination in the treatment of well-differentiated neuroendocrine tumors. Onco Targets Ther. 2016 Feb;9:699-704.

29 Frilling A, Li J, Malamutmann E, Schmid KW, Bockisch A, Broelsch CE. Treatment of liver metastases from neuroendocrine tumours in relation to the extent of hepatic disease. $\mathrm{Br} \mathrm{J}$ Surg. 2009 Feb;96(2):175-84.

30 Sorbye H, Welin S, Langer SW, Vestermark LW, Holt N, Osterlund P, et al. Predictive and prognostic factors for treatment and survival in 305 patients with advanced gastrointestinal neuroendocrine carcinoma (WHO G3): the NORDIC NEC study. Ann Oncol. 2013 Jan; 24(1):152-60.

31 Kos-Kudła B, Blicharz-Dorniak J, Strzelczyk J, Bałdys-Waligórska A, Bednarczuk T, Bolanowski $\mathrm{M}$, et al. Diagnostic and therapeutic guidelines for gastro-entero-pancreatic neuroendocrine neoplasms (recommended by the Polish Network of Neuroendocrine Tumours). Endokrynol Pol. 2017;68(2):79-110.

32 Eisenhauer EA, Therasse P, Bogaerts J, Schwartz LH, Sargent D, Ford R, et al. New response evaluation criteria in solid tumours: revised RECIST guideline (version 1.1). Eur J Cancer. 2009 Jan;45(2):228-47.

33 National Cancer Institute. Common Terminology Criteria for Adverse future Events. Common terminology criteria for adverse events v5.0 (CTCAE). Cancer Ther Eval Progr 2017. Available from: http://ctep.cancer.gov.

34 von Elm E, Altman DG, Egger M, Pocock SJ, Gøtzsche PC, Vandenbroucke JP; STROBE Initiative. The Strengthening the Reporting of Observational Studies in Epidemiology (STROBE) statement: guidelines for reporting observational studies. Lancet. 2007 Oct; 370(9596):1453-7.
35 Villano JL, Collins CA, Manasanch EE, Ramaprasad C, van Besien K. Aplastic anaemia in patient with glioblastoma multiforme treated with temozolomide. Lancet Oncol. 2006 May;7(5):436-8.

36 Noronha V, Berliner N, Ballen KK, Lacy J, Kracher J, Baehring J, et al. Treatment-related myelodysplasia/AML in a patient with a history of breast cancer and an oligodendroglioma treated with temozolomide: case study and review of the literature. Neuro Oncol. 2006 Jul;8(3):280-3.

37 Jalali R, Singh P, Menon H, Gujral S. Unexpected case of aplastic anemia in a patient with glioblastoma multiforme treated with Temozolomide. J Neurooncol. 2007 Oct; 85(1):105-7.

38 Saif MW, Kaley K, Brennan M, Garcon MC, Rodriguez $\mathrm{G}$, Rodriguez T. A retrospective study of capecitabine/temozolomide (CAPTEM) regimen in the treatment of metastatic pancreatic neuroendocrine tumors (pNETs) after failing previous therapy. JOP. 2013 Sep;14(5):498-501.

39 Crespo G, Jiménez-Fonseca P, Custodio A, López C, Carmona-Bayonas A, Alonso V, et al. Capecitabine and temozolomide in grade 1/2 neuroendocrine tumors: a Spanish multicenter experience. Future Oncol. 2017 Mar; 13(7):615-24.

40 Strosberg JR, Cives M, Brelsford M, Black M, Meeker A, Ghayouri M. Identification of response predictors to capecitabine/temozolomide in metastatic pancreatic neuroendocrine tumors. J Clin Oncol. 2015;33(15_suppl):4099.

41 Abdel-Rahman O, Fouad M. Temozolomidebased combination for advanced neuroendocrine neoplasms: a systematic review of the literature. Future Oncol. 2015;11(8):127590.

42 Schmitt AM, Pavel M, Rudolph T, Dawson $\mathrm{H}$, Blank A, Komminoth P, et al. Prognostic and predictive roles of MGMT protein expression and promoter methylation in sporadic pancreatic neuroendocrine neoplasms. Neuroendocrinology. 2014;100(1): 35-44.

43 Cros J, Hentic O, Rebours V, Zappa M, Gille $\mathrm{N}$, Theou-Anton N, et al. MGMT expression predicts response to temozolomide in pancreatic neuroendocrine tumors. Endocr Relat Cancer. 2016 Aug;23(8):625-33.

44 Reidy DL, Basturk O, Kriplani A, Abou-Alfa GK, Jarnagin WR, Allen PJ, et al. MGMT immunohistochemistry (IHC) and exclusion of pancreatic NET (PanNET) patients from treatment with temozolomide-based therapy. J Clin Oncol. 2014;32(15_suppl):e15169-15169. 